\title{
WERKGESCHICHTE OHNE WERK: ORHAN PAMUKS SCHNEE AUS DEKONSTRUKTIVER PERSPEKTIVE
}

\section{Onur Kemal BAZARKAYA ${ }^{1}$}

\begin{abstract}
Öz: Yapı bozumculuk, Orhan Pamuk'un Kar isimli eseriyle ilgili araştırmalarda bugüne kadar yöntemsel olarak neredeyse hiç rol oynamamıştır. Romanın kendisi aslen kesin bir şekilde yapı bozumcu bir işleve sahip olduğundan bu durum oldukça kayda değerdir. $\mathrm{Bu}$ yazıda Jacques Derrida'ya benzer bir okuma yöntemi geliştirilmektedir. Önce Derrida'nın kapalı metinlerden değil, bilakis "farklılıklar ve izlerin izleri" gibi evrensel metin anlayışından yola çıkan kavramlar hakkında bilgi verilip, bu görüş Kar-Mecazı ve Franz Kafka'nın Şato adlı eseri bağlamında örneklendirilmektedir. Bu bağlamda ayrıca son bahsedilen ile tüm anlamsal varoluşa ilişkin engellenen "différance" kavramı incelenmektedir. Sonrasında çalışma, Kar'daki genç kadınların arasında baş gösteren karmaşık bir başörtüsü sorunsalı ile bağlantılı "intihar salgını"nı ele alarak devam etmektedir. Romanın kahramanı Ka, nafile bir şekilde intiharların sebeplerini araştırmayı denemektedir. Çünkü görüleceği üzere, her bir olayın ardında "différance"a uygun bir şekilde gelişen kendine özgü anlayış değişkeleri yatmaktadır. Yapı bozumcu bir eser hikâyesini, geliştirilen tezin son bölümünde olduğu gibi bir eser olmadan anlatan anlatıcı Orhan, kendi içinde bir problemi de Anlamak olgusunu ortaya koymaktadır. Duruma bu yönden bakıldığında Pamuk'un romanının büyük bölümünü ele alan Ka'nın Kar'ı adlı şiir kitabı da meçhuldür. Fakat anlatıcı, şiir kitabı hakkındaki bilgileri asıl kitabın yerine geçirerek, Pamuk'un Kar'ını zorunlu olarak eserin oluşumuna katkı sağlayan sosyal süreçte ortaya koymuştur.
\end{abstract}

Anahtar Sözcükler: Orhan Pamuk, Kar, Franz Kafka, Yapı Bozumculuk, Différance, Anlamak, Eser Hikâyesi.

\section{Einleitung: Spuren nach Kars}

Die nordostanatolische Provinzstadt Kars wird in Orhan Pamuks Roman Schnee $($ Kar , 2002) als ein Ort beschrieben, der gleichsam aus der Zeit gefallen ist. Es ist die Rede davon, dass ihn der Staat und Allah vergessen hätten (,devletin ve Allah'1n Kars'1 unutmas1“, Pamuk, 2002, S. 30). Wer hier lebe, heißt es, scheine nicht wirklich zu existieren und wolle entweder sterben oder verschwinden (ebd. S. 56). „Zieh die Spülung, mach Schluß, wenn's nach Kars dich

\footnotetext{
${ }^{1}$ Yrd. Doç. Dr., Namık Kemal Üniversitesi, Fen-Edebiyat Fakültesi, Alman Dili ve Edebiyatı Bölümü. okbazarkaya@nku.edu.tr
} 
verschlägt!“‘2 („Çek sifonu gitsin kişi düştüyse şehri Kars’a“, Pamuk, 2002, S. $105)$ heißt es in einem Gedicht, das ein Verzweifelter an die Wand eines Karser Teehauses geschrieben hat.

$\mathrm{Zu}$ dieser verlorenen Stadt verhält sich der Protagonist in gewisser Weise analog. Kerim Alakuşoğlu, Ka genannt, wuchs im reichen Istanbuler Stadtteil Nişantaş1 in gutbürgerlichen Verhältnissen auf. Als Dichter erwarb er sich einiges Ansehen. Nach dem Militärputsch 1980 wurde er aufgrund eines politischen Artikels, den er nicht selbst verfasst hatte, verurteilt und floh deshalb ins Exil nach Frankfurt, wo er völlig vereinsamt von Sozialhilfe und seinen Lesungen lebt. Bei Ka handelt es sich, kurz gesagt, um einen sozial Entwurzelten. Der Tod seiner Mutter veranlasst ihn dazu, zum ersten Mal seit zwölf Jahren nach Istanbul zurückzukehren. Nach einem viertägigen Aufenthalt in seiner Heimatstadt bricht er auf zu einer Reise nach Kars.

Einer der Gründe für Pamuks Wahl dieser Stadt als Haupthandlungsort des Romans ist Michael McGaha zufolge der Umstand, dass in ihrem Namen der Begriff Schnee (,Kar") enthalten ist:

Part of the citiy's attraction for Pamuk was surely its very poetic name; Kars is a shortened version of the Turkish Kar-su (,Snow Water"). He originally intended to title his novel Kars but later, concerned that it might be mistaken for a guidebook or a history of the city, changed it to Kar. The later title was more appropriate anyway, because snow is the novel's central, all-pervasive metaphor (McGaha, 2008, S. 158).

Als „all-pervasive metaphor“ erfüllt „Schnee“ im Text tatsächlich mehrere Funktionen: Es tritt (1.) als meteorologisches Phänomen auf, in dessen Beschreibungen sich die komplizierte Psyche des Protagonisten je nach Stimmungslage widerspiegelt. "Schnee“ heißen (2.) sowohl der hier zu untersuchende Roman (von dem auch im Text die Rede ist) als auch (3.) Kas in Kars entstandener Gedichtzyklus einschließlich das Titelgedicht. Nicht zuletzt kann „Schnee“, auch in Kombination mit anderen Begriffen oder Metaphern, (4.) bestimmte literarische Assoziationen wecken, die aber, wie weiter unten zu sehen sein wird, gleichsam im Text angelegt sind.

$\mathrm{Zu}$ nennen wäre hier beispielsweise das Schnee-Kapitel in Thomas Manns Der Zauberberg (1924). Darin hat Hans Castorp bekanntlich inmitten eines Schneesturms einen Traum, in dem er die vorläufige „Summe seiner hermetischen Bildungserfahrungen“ (Karthaus, 1983, S. 100) zieht; „Der Mensch soll um der Güte und Liebe willen dem Tode keine Herrschaft einräumen über seine Gedanken“ (Mann, 1960, S. 686) lautet der berühmte Satz, in dem sie sich widerspiegelt. Nun geht nach einer verbreiteten Ansicht in der Mann-Forschung die verführerische Macht, die Eros und Thanatos von Beginn an auf den Protagonisten ausüben, infolge dieser Episode keineswegs zurück. ${ }^{3}$ Hans Castorps Todessehnsucht teilen auch viele Figuren in Pamuks

\footnotetext{
${ }^{2}$ Der vorliegende Beitrag hält sich an die Übersetzung von Neumann 2005.

${ }^{3}$ Zur Forschungsdiskussion vgl. Koopmann, 1983, S. 49ff., vgl. auch Schmidt, 1997, S.165-174,
} 
Schnee, so etwa die jungen Frauen, die sich aus letztlich unerklärlichen Gründen das Leben genommen haben. Der Slogan der Karser Antiselbstmordkampagne - „Der Mensch ist ein Meisterwerk Gottes, und Selbstmord ist Gotteslästerung“ (,İnsan Allah'ın Bir Şaheseridir ve İntihar Bir Küfürdür“, Pamuk, 2002, S. 13) - klingt wie eine Übertragung der zentralen Worte aus Hans Castorps Schnee-Traum in den islamisch-türkischen Kontext. ${ }^{4}$

„Schnee“ ist in Pamuks Roman nicht gleichbedeutend mit „Schnee“. Das Zeichen kann vieles - und vieles gleichzeitig - meinen. Durch den Namen des Protagonisten, Ka, der semiotisch sowohl in Kar (Schnee) als auch im Stadtnamen Kars enthalten ist, wird die Mehrdeutigkeit zusätzlich erhöht. Bei allen semantischen Überlagerungen, die hier entstehen können, bildet das $\mathrm{K}$ gewissermaßen die kleinste semiotische Einheit. Als solche tritt es im Übrigen nur einmal buchstäblich in Erscheinung, und zwar dann, wenn der auf Kas Spuren wandelnde Orhan erzählt, dass just an der Stelle, wo sein Freund einst erschossen worden war, ein Neon-K ,aus dem Aschgrau des hervorbrechenden Abends rosa hervorleuchtete" (,çökmekte olan akşamin kül rengi içinde pırıl pirıl bir pembeyle parlayan neon bir K harfi[]“, ebd. S. 253). Insgesamt lässt sich im Hinblick auf die in der Buchstabenfolge $\mathrm{K}-\mathrm{a}-\mathrm{r}-\mathrm{s}$ enthaltenen Zeichenmöglichkeiten mit Jacques Derrida von einem komplexen „Spiel der Differenzen" sprechen, das

Synthesen und Verweise voraus[setzt], die es verbieten, daß zu irgendeinem Zeitpunkt, in irgendeinem Sinn, ein einfaches Element als solches präsent wäre und nur auf sich selbst verwiese. Kein Element kann je die Funktion eines Zeichens haben, ohne auf ein anderes Element, das selbst nicht präsent ist, zu verweisen, sei es auf dem Gebiet der gesprochenen oder auf dem der geschriebenen Sprache. Aus dieser Verkettung folgt, daß sich jedes „Element“ Phonem oder Graphem - aufgrund der in ihm vorhandenen Spur der anderen Elemente der Kette oder des Systems konstituiert. Diese Verkettung, dieses Gewebe ist der Text, welcher nur aus der Transformation eines anderen Textes hervorgeht. Es gibt nichts, weder in den Elementen noch im System, das irgendwann oder irgendwo einfach anwesend oder abwesend wäre. Es gibt durch und durch nur Differenzen und Spuren von Spuren (Derrida 1990, S. 150-151).

Beim „Schnee“ handelt es sich so gesehen um eine „Spur“, die selbst wiederum „Spuren von Spuren“ enthält. Neben der anhand des Traums von Hans Castorp veranschaulichten Schneemetapher bildet im Pamuk'schen Text der Name Ka von allen „Spuren“ gleichsam die spurenreichste. Wie unschwer zu erkennen ist, liegt mit ihm ein Bezug zu den Romanen Der Proceß (1914/1915) und Das Schloß (1922) von Franz Kafka vor, deren Protagonisten beide K. heißen. Außer den differentiellen Verweisen auf „Schnee“ bzw. die Stadt Kars verdichten sich im Namen Ka also auch solche auf diese Kafka'schen Werke. Besonders

vgl. auch Mennicken, 2002, S. $103 \mathrm{ff}$.

4 Für weitere literarische Beispiele von Schnee-Metaphern, die in Pamuks Darstellung metaphorologisch aufgehoben sein können, siehe kursorisch Göknar, 2013, S. 198. In diesem Zusammenhang vgl. auch Blumenberg 1997. 
ausgeprägt sind dabei die Anspielungen auf den Schloß-Roman, ${ }^{5}$ der folgendermaßen beginnt: „Es war spät abend als K. ankam. Das Dorf lag in tiefem Schnee. Vom Schloßberg war nichts zu sehn, Nebel und Finsternis umgaben ihn, auch nicht der schwächste Lichtschein deutete das große Schloß an. Lange stand K. auf der Holzbrücke die von der Landstraße zum Dorf führt und blickte in die scheinbare Leere empor (Kafka, 1995, S. 9).“ Ähnlich wird im Eingangskapitel von Schnee die Ankunft des Protagonisten an seinem Reiseziel beschrieben: „Als der Bus mit drei Stunden Verspätung um zehn Uhr abends in die schneebedeckten Straßen von Kars einbog, erkannte Ka die Stadt überhaupt nicht wieder. [...] Unter dem Schnee war alles wie ausgelöscht, wie verloren“ („Otobüs karlar altındaki Kars sokaklarına saat onda, üç saat gecikmiş olarak girdiğinde Ka şehri hiç tanıyamadı. [...] Karin altında her şey silinmiş, kaybolmuş gibiydi“", Pamuk, 2002, S. 12). In beiden Zitaten erreicht der Protagonist seinen Bestimmungsort erst zu später Stunde. K. richtet den Blick „in die scheinbare Leere empor", wo das rätselhafte Schloss steht, das er trotz aller Bemühungen niemals erreichen wird; seine Mission als Landvermesser ist zum Scheitern verurteilt. Auch Ka wird in seinem Vorhaben scheitern. Offiziell ist er im Auftrag der Zeitung Cumhuriyet nach Kars gereist, um über die Lokalwahlen und die sich wie eine Epidemie ausbreitenden Selbstmorde junger Frauen zu berichten. Am nächsten Tag wird ihm jedoch der wahre Grund seiner Reise bewusst: Er ist gekommen, um seine in Kars lebende, ehemalige Kommilitonin İpek zu heiraten. Max Brod zufolge ist es hauptsächlich der „Kampf um Liebe und um Eingliederung in die Gesellschaft" (Brod, 1968, S. 303), die den Protagonisten von Das Schlo $\beta$ umtreibt, ein Handlungsmotiv, das auch auf den sozial entwurzelten Ka zutrifft. Doch diesem ist die Erfüllung seines Heiratswunsches ebenso wenig beschieden wie die Wahrheit über die Selbstmorde.

Wie das Schloss für K. unerreichbar bleibt, so entzieht es sich tendenziell der Sinnbestimmung durch den Leser. ${ }^{6}$ Insofern gleicht es der Wahrheit von Kafkas Proce $\beta$, die ,[e]ndlos verschoben“ wird, indem man ,mit dem Protagonisten K. von Deutung zu Deutung eilt, ohne jemals die wahre Bedeutung zu finden“ (Zima, 1994, S. 66). Differentielle Sinnverschiebungen dieser Art, für die Derrida den Begriff der ,différance " geprägt hat, vereiteln jegliche Sinnpräsenz: „Der Sinn kann nie gegenwärtig sein, weil er sich in einem stets offenen Verweisungszusammenhang verschiebt und dadurch einem Bedeutungswandel unterliegt“" (ebd. S. 52). Unter Bezugnahme auf Derridas Interpretation von Der Proce $\beta$ meint Peter V. Zima, dass die darin enthaltene Parabel Vor dem Gesetz in gewisser Weise als „Erzählung der différance“ (ebd. 65) und mithin als „Allegorie der Dekonstruktion“ (ebd. S. 66) gelesen werden kann. Nicht zuletzt

\footnotetext{
${ }^{5}$ Dies hat 2004 bereits John Updike in seinen Anatolian Arabesques festgestellt (McGaha, 2008, S. 158).

${ }^{6}$ Hans H. Hiebel spricht hier in Anlehnung an Gerhard Neumanns Terminus des ,gleitenden Paradoxons“ von einer ,gleitenden Metapher“ (Hiebel, 1999, S. 15 ff).
} 
aufgrund seiner Kafka-,Spur“ lässt sich diese Lesart m. E. auch auf Pamuks Roman veranschlagen. Schnee ist gleichsam von einer dekonstruktiven Bewegung gekennzeichnet, für die im Folgenden - wiederum mit Mitteln der Dekonstruktion - eine entsprechende Lesart entwickelt werden soll. ${ }^{7}$

\section{Rätselhafte Spuren: Die „Selbstmordepidemie“}

$\mathrm{Zu}$ Beginn seines Aufenthalts in Kars geht Ka der mysteriösen Serie von Selbstmorden junger Frauen nach. Von einem solchen Fall war zuerst die Hunderte von Kilometern entfernte Stadt Batman betroffen. In Kars brach die „Selbstmordepidemie“ (,intihar salgını[]“, Pamuk, 2002, S. 20) aus, als die Medien im In- und Ausland ausführlich davon berichteten, weshalb der stellvertretende Gouverneur Ka denn auch bittet, die Sache in der Cumhuriyet vorsichtshalber nicht hochzuspielen.

Als Ursache für die Suizide erblickt man im Allgemeinen die patriarchalischen Strukturen der türkischen Gesellschaft. Schläge von Vätern und Ehemännern, Verführung durch Verheiratete, Denunziation durch Autoritätspersonen, männliche Gewalt und Unterdrückung allenthalben hätten die Frauen zum Selbstmord getrieben. Wie einer Kafka'schen Romanfigur bleibt $\mathrm{Ka}$ die Wahrheit jedoch verborgen. „Je mehr ich es [Kars] kennenlerne“, gesteht er Kadife, „um so mehr habe ich das Gefühl, keinem außerhalb erklären zu können, was hier passiert“ („Tanıdıkça da burada olup bitenleri dışarıda kimseye anlatamayacağımı hissediyorum“, ebd. S. 114). Pauschale Urteile wie etwa das des stellvertretenden Gouverneurs, der vom „Gefühl extremen Unglücks“ bei den Selbstmörderinnen spricht („,bu kızlarımızın aşırı mutsuzluğu“, ebd. S. 20), oder das Handes, die den Wunsch nach „Kontrolle über den eigenen Körper“" (,kendi vücudumuza sahip olmak“, ebd. S. 125) beschwört, helfen ihm nicht weiter. Umgekehrt erweisen sich manche Erklärungen bei genauerer Prüfung als widersprüchlich, so etwa die für den Tod Teslimes, jener von der säkularen Presse als „Turban-Mädchen“ (,türbancı k1z“, ebd. S. 22) bezeichneten Studentin, die sich mit ihrem Kopftuch erhängt hat. Da sie dieses auch als Symbol des politischen Islam verstand, sehen viele in ihrer Tat einen Protest gegen das strikte Kopftuchverbot an ihrer Hochschule, in die ihr nach anfänglichem Unterrichtsausschluss der Zutritt ganz verweigert wurde. Mit dieser Auffassung divergiert indes der Umstand, dass der Selbstmord im Islam eine Sünde ist und Teslime deshalb als Ungläubige starb. Teilweise wird sogar gemutmaßt, dass sie insgeheim eine Atheistin gewesen sei, da sie sich sonst nicht umgebracht hätte. Schließlich kursiert die vom Nationalen Nachrichtendienst in Umlauf gebrachte Version, der zufolge ihr Selbstmord

\footnotetext{
${ }^{7}$ Eine dekonstruktive Untersuchung von Schnee hat, wie es aussieht, bislang nur Sibel Erol vorgenommen, der zufolge Pamuks vielberufenes Spiel mit den Begriffen Ost und West lediglich das Verhältnis von Gleichheit und Differenz im Sinne Derridas widerspiegelt. Differenzen dekonstruktiv zu relativieren und aufzuheben, ist ihrer Ansicht nach das primäre poetische Ziel des Autors (Erol, 2007, S. 403). Erols verdienstvolle Arbeit hat der Pamuk-Forschung einen Bereich eröffnet, in den sich der vorliegende Beitrag einordnet.
} 
darauf zurückzuführen ist, dass sie ihre Unschuld an einen bereits verheirateten Polizisten verlor.

Was Ka im Falle Teslimes das Finden der Selbstmordursache vollends unmöglich macht, ist das Kopftuchthema ${ }^{8}$ von dessen Komplexität ihm Kadife einen lebhaften Eindruck vermittelt. Sie erzählt ihm von der Zeit, als sie begann, ein Kopftuch zu tragen. Ihr Vater, ein alter Kommunist, der für seine politischen Überzeugungen im Gefängnis saß, reagierte darauf mit zutiefst gespaltenen Gefühlen:

Babam başta benimle iftihar etti, başımı örtüp okula gittiğim gün bu çok özel bir başkaldırı usulüymüş gibi davrandı. Benimle birlikte annemden kalma pirinç çerçeveli aynaya bakarak başörtümün başımın üzerinde duruşunu seyretti ve biz aynanın karşısındayken öptü beni. Aramızda çok az konuşulmasına rağmen şurası kesindi: Benim yaptığım İslamcı bir hareket olduğu için değil, devlete karşı bir hareket olduğu için saygıdeğerdi. Babamda 'benim kızıma böylesi yakışır' havası vardı, ama gizliden gizliye o da benim gibi korkuyordu. Bizi içeri aldıklarında korktuğunu, pişman olduğunu biliyorum. Siyasi polisin benimle değil hâlâ kendisiyle uğraştığını ileri sürdü. Bir zamanlar buralarda harıl harıl solcuları, demokratları fişleyen MİT elemanları, şimdi dincilerin çetelesini tutuyordu; işe eski tüfeğin kızından başlamaları anlaşılır bir şeydi filan. Bütün bunlar benim geri adım atmamı zorlaştırıyor, babam da benim her adımıma destek vermek zorunda kalıyordu, ama gittikçe zorlaşıyordu bu. [...] İntikamını o kızlardan biri bazen eve gelirse ona hınzırca ateistlik ederek alıyor, ama işler sonunda o kızlarla bir çeşit devlet karşıtı cilveleşmeye dönüşüyor. Kızların da altta kalmadan babama laf yetiştirebilmelerini bir olgunluk olarak gördüğüm için evde toplantılar yapiyorum (Pamuk, 2002, S. 116).

Am Anfang war mein Vater stolz auf mich; an dem Tag, an dem ich mit bedecktem Haupt in die Uni ging, hat er sich benommen, als sei das eine besondere Form der Revolte. Er hat sich in dem Messingspiegel, der noch von unserer Mutter stammt, zusammen mit mir angeschaut, wie das Tuch auf meinem Kopf aussah, und mir vor dem Spiegel einen Kuß gegeben. Auch wenn wir kaum darüber sprechen, ist eines klar: Was ich tat, verdiente Hochachtung, nicht weil es eine islamistische Tat war, sondern weil es sich gegen den Staat richtete. Mein Vater nahm die Haltung ein: So etwas ist ganz meine Tochter, aber insgeheim hat er sich genauso gefürchtet wie ich. Ich weiß, daß er Angst gehabt hat, als sie uns eingesackt haben, daß er Gewissenbisse hatte. Er behauptete, daß die politische Polizei nicht hinter mir, sondern immer noch hinter ihm her sei. Die Angehörigen des Nationalen Nachrichtendienstes, die früher einmal fieberhaft die Linken, die Demokraten bespitzelt hätten, überwachten jetzt die Religiösen; es sei ganz verständlich, daß sie mit der Tochter eines alten Kämpfers beginnen und so weiter. Deswegen wurde es für mich schwieriger, einen Rückzieher zu machen, und mein Vater war gezwungen, mich bei jedem meiner Schritte zu unterstützen, aber das wurde immer komplizierter. [...] Wenn manchmal eines von den Mädchen zu uns nach Hause kommt, rächt er sich, indem er sich wie ein gemeines Schwein als Atheist aufführt, aber am Ende mündet das in eine Art Anti-Staat-Stichelei mit diesen

\footnotetext{
${ }^{8}$ Vgl. hierzu Neumann 2011, Clemens 2011 und Chang 2002.
} 
Mädchen. Ich veranstalte zu Hause Treffen, weil ich es als ein Zeichen der Reife sehe, wenn die Mädchen da nicht schweigen, sondern bei meinem Vater dagegenhalten.

Im Zitat fungiert das Kopftuch als semiotisches Zeichen, dessen Bedeutung sich in den jeweiligen politischen Kontexten verschiebt und so in seiner ,différance sichtbar wird. Ironisch bringt Kadife den Narzissmus ihres Vaters, eines stolzen Staatsgegners, zur Sprache, der sich in ihrer Entscheidung, sich das Haupt zu bedecken, buchstäblich bespiegelt. Wie ihre verstorbene Freundin Teslime ist sie eine Anhängerin des politischen Islam und versteht das Kopftuch deshalb auch als Symbol des Widerstands gegen den laizistischen Staat. Insofern stimmt ihr Vater, der das Kopftuchtragen bei ihr als „,besondere Form der Revolte“ (,,çok özel bir başkaldırı usulüymüş“) begrüßt, mit ihr überein. Doch bleibt die Kopfbedeckung seiner Tochter, was sie in erster Linie ist, nämlich ein Ausdruck ihres religiösen Glaubens, den er als Kommunist bzw. Atheist ablehnt. Je nach Kontext bedeutet das Kopftuch also Widerstand gegen den staatlichen Machtapparat, sei es (1.) im Zeichen des politischen Islam oder (2.) im Zeichen allgemeinen Protests, und (3.) Glaubenssymbol. Über das Zitat hinaus wird es im Roman ferner apostrophiert als (4.) islamisch-patriarchales Mittel der Unterdrückung.

Angesichts dieser ihm gleichsam eingeschriebenen Vieldeutigkeit erscheint das Kopftuch, mit dem sich Teslime erhängt hat, als Chiffre der Unzugänglichkeit. Seine Botschaft kann vor dem Hintergrund des bereits erwähnten Kopftuchverbots an der Hochschule im Sinne politischer Unbeugsamkeit gedeutet werden. Dagegen spricht jedoch der gewaltsame Akt des Sicherhängens als solcher: Dass Teslime ihr Kopftuch als Strick benutzt hat, kann darauf verweisen, dass sie dieses Mittel ihrer Unterdrückung, als das sie es in ihrem Innersten empfunden haben mag, für ihren Freitod in autoaggressiver Umkehrung nutzte. Weitere Deutungsmöglichkeiten ließen sich kombinieren; die Wahrheit bleibt jedoch in jedem Fall verborgen.

In seiner Rätselhaftigkeit ist Teslimes Tod für die Selbstmorde, nach deren Ursachen Ka vergeblich forscht, symptomatisch. Jeder dieser Suizide steht in gewisser Weise für das letztlich unzugängliche Schicksal des ,Anderen'. Gemeint ist hier keine Alterität im interkulturellen Sinne, die sich etwa auf die vielberufene Ost-West-Dichotomie im Roman beziehen würde, sondern vielmehr eine solche, die existenzialistische Inhalte akzentuiert. ${ }^{9}$ Die Identifikation mit dem ,Anderen' bestimmte Pamuk in seiner Dankesrede zum Friedenspreis des deutschen Buchhandels 2005 als ureigene Aufgabe des Romanschriftstellers, dem es vorbehalten sei, ,genau andersherum zu denken, als es der allgemeinen Erwartung entspricht" (Pamuk, 2005, S. 27). Gemäß dieser Ansicht setzt sich der ,autodiegetische“, d. h. auch als Protagonist auftretende Erzähler (Genette, 1998, S. 201 f.) von Schnee - der im Übrigen auch denselben Vornamen trägt wie der Autor und sich als Romancier

\footnotetext{
${ }^{9}$ Vgl. hierzu auch Afridi und Buyze, 2012.
} 
bezeichnet - mit dem ,Anderen' auseinander. An einer Stelle, von der er behauptet, dass sie wohl „das Herz unserer Geschichte“ sei („Belki de hikâyemizin kalbine geldik“, Pamuk, 2002, S. 259), befasst sich Orhan mit folgenden Fragen:

Başkasının acısını, aşkını anlamak ne kadar mümkündür? Bizden daha derin acılar, yokluklar, eziklikler içinde yaşayanları ne kadar anlayabiliriz? Anlamak eğer kendimizi bizden farklı olanın yerine koyabilmekse dünyanın zenginleri, hakimleri, kenarlardaki milyarlarca garibanı hiç anlayabildiler mi? Romancı Orhan, şair arkadaşının zor ve acı hayatındaki karanlı̆̆ı ne kadar görebilir? (ebd.) Wie weit ist es möglich, den Schmerz und die Liebe eines anderen zu verstehen? Wie weit können wir diejenigen verstehen, die schlimmere Qualen, Nöte und Verletzungen durchleben als wir? Wenn Verstehen heißt, sich an die Stelle eines von uns Verschiedenen zu versetzen, haben dann die Reichen und Mächtigen der Welt jemals die Milliarden armer Schlucker je verstehen können? Wie viel von der Dunkelheit im schwierigen und leidvollen Leben seines Dichterfreundes kann der Romancier Orhan durchdringen?

Der Erzähler wirft hier ein existenzielles Schlaglicht auf die erniedrigten und beleidigten ,Anderen' in der Gesellschaft und thematisiert das Verstehen ihres Leides, das unter Umständen schwerer wiegt als das eigene. Seine Fragen nach dem „Wie weit" und „Wie viel“" (,ne kadar") verraten indes seine Zweifel daran, dass es sich restlos begreifen lässt. Das Zitat handelt also von den Grenzen gegenseitigen Verstehens, deren Hintergrund von der weiter oben illustrierten „différance“ gebildet wird. In der rhetorischen Frage, ob die gesellschaftlichen Eliten die unterprivilegierten Schichten je verstanden hätten, klingt zudem eine unterschwellige Kritik an den politischen Strukturen an, auf die das Auseinanderdriften von Arm und Reich letztlich zurückgeht.

\section{Auf Kas Spuren wandelnd: Orhans stellvertretende Autorschaft}

Für das Zitat bietet sich noch eine weitere, methodische Lesart an. Auf ihrer Grundlage verweisen die Grenzen gegenseitigen Verstehens auf jene der Hermeneutik, deren Ansatz sich grundsätzlich von dem der Dekonstruktion unterscheidet. ${ }^{10}$ Schnee ist in gewisser Weise eine fiktionale Werkgeschichte ohne Werk, denn Kas gleichnamiger Gedichtzyklus, der zentrale Gegenstand des Romans, ist nach seinem Tod nicht auffindbar. Orhan bereist in der Rahmenhandlung die letzten Lebensstationen seines Freundes und sammelt „mit der Leidenschaft eines Museologen“ (,bir müzeci aşkılla“, Pamuk, 2002, S. 258) alles, was nur irgendwie mit Ka zusammenhängt. Da es ihm besonders um die Gedichte zu tun ist, sucht er Kas Frankfurter Wohnung auf, muss dort aber feststellen, dass das grüne Heft, von dem er weiß, dass es sie enthält, verloren ging oder gestohlen wurde. Trotzdem reist er auf den Spuren des Verstorbenen weiter nach Istanbul und von dort aus schließlich mit dem Bus nach Kars, wo er, wie $\mathrm{Ka}$ einst, an einem Winterabend ankommt, sich im selben Hotel einquartiert, im Schnee dieselben Bürgersteige entlanggeht, mit denselben

\footnotetext{
${ }^{10}$ Zur wissenschaftlichen Kontroverse beider Richtungen vgl. exemplarisch Tholen, 1999.
} 
Leuten spricht und sich sogar in dieselbe Frau verliebt (ebd. S. 381 ff.). Vom Vorgehen her wäre das Hermeneutik par excellence, ${ }^{11}$ fehlte Orhan hierfür nicht das Werk und stellte sich ihm, dem angesichts der „différance" zweifelnden Hermeneutiker, nicht die Frage, inwieweit (,ne kadar") er den Autor überhaupt verstehen könne.

Durch eine dekonstruktive Inversion (Culler, 1988, S. 177 ff.), in diesem Fall also die Konzentration auf marginale Informationen über das Werk statt auf das Werk selbst, kann er trotzdem vom Gedichtzyklus erzählen. Die Voraussetzung für ein solches Verfahren bildet die weiter oben mit Derrida referierte Leugnung eines Textäußeren, wie sie für den Dekonstruktivismus kennzeichnend ist. Der Fokus dieser Methode liegt, wie gesehen, auf der universalen Geschlossenheit von Texten bzw. ihren „Differenzen und Spuren von Spuren“. Orhans aus dem Geist des Dekonstruktivismus entstandene Werkgeschichte ohne Werk entspricht dem real existierenden Roman Schnee, der sich bekanntlich zu einem Großteil aus den Inhalten der von Ka hinterlassenen Hefte speist, in denen der Dichter seine Erlebnisse in Kars und die gleichsam daraus resultierenden Verse kommentiert. Unter seinen Aufzeichnungen findet sich auch die beschriftete Figur eines in die Achsen der Vernunft, der Phantasie und der Erinnerung aufgeteilten Schneekristalls, aus dem Orhans Ansicht nach die „tiefe und geheime' Ordnung“ (,„derin ve esrarlı' bir düzeni“, Pamuk, 2002, S. 257) der Ka'schen Gedichte hervorgeht. So dient der Schneekristall dem dekonstruktiven Werkbiografen zur Orientierung.

Ka ist, wie in Pamuks Vorstellung die Lyriker im Allgemeinen (McGaha, 2008, S. 161), in hohem Maße begeisterungsfähig und voller Inspiration. Verglichen mit diesem „wahre[n] Dichter, der ganz so lebte, wie es ihm ganz entsprach“ (,içinden geldiği gibi, kendi olarak yaşayabilen gerçek bir şair“, Pamuk, 2002, S. 414-415), fühlt sich Orhan wie ,ein Romanschriftsteller mit einer relativ simplen Seele, der jeden Morgen und jede Nacht zu bestimmten Stunden wie ein Sekretär arbeitet" (,her sabah, her gece belirli saatlerde bir kâtip gibi çalışan daha basit ruhlu bir romanc1“, ebd. S. 415). In Kars flossen die Eindrücke, die der begeisterte Ka von seiner Umgebung gewann, nahezu unmittelbar in seine Gedichte ein. Dass er sie später in seinen Kommentaren einzeln wieder aufgriff und erläuterte, ist ein für Orhan vorteilhafter Umstand: Von ihnen ausgehend, kann er die Werkgeschichte ohne Werk erzählen.

Die Konzentration des ,autodiegetischen“ Erzählers von Schnee auf vermeintlich marginale Einzelheiten entspricht der inneren Überzeugung des Autors. „Wissen Sie“, bekannte Pamuk in einem Interview mit Jörg Lau,

\footnotetext{
${ }^{11}$ Eine genaue Einordnung der Hermeneutik, die Orhans Recherchen in Ansätzen zugrunde liegt, ist für den Fortgang dieser Arbeit nicht notwendig und würde ihren Rahmen sprengen. Vermutlich handelt es sich hier um ein eklektizistisches Verfahren, bei dem die gesamte Lebenssituation des Autors mittels der Einfühlung und unter Einbeziehung aller Formen seiner Äußerungen (Schleiermacher/Dilthey) ebenso berücksichtigt wird wie die existenziale und geschichtliche Dimension des Verstehens (Heidegger/Gadamer). Vgl. hierzu exemplarisch Birus, 1982.
} 
ich habe die großen Ideen gründlich satt. Ich bin ihnen in meinem überpolitisierten Land viel $\mathrm{zu}$ sehr ausgesetzt gewesen. Literatur ist meine Reaktion darauf, ein Versuch, das Spiel umzudrehen, einen gewissen Humor, eine gewisse Distanz in die Sache zu bringen. Ich will dem Leser sagen: Nimm diese Dinge nicht so verdammt wichtig. Ist das Leben nicht schön? Achte auf die Details des Lebens! Das Wesentliche im Leben ist Glück und ist die Möglichkeit, in dieser intoleranten Gesellschaft, die wir geschaffen haben, zu überleben. (Lau, 2005, S. 4)

Gemäß dieser Einstellung versucht Orhan, „das Spiel umzudrehen“, und rückt anstelle des Werks selbst „,die Details des Lebens“, die Ka zu seinen Gedichten inspirierten, ins Zentrum seiner Werkgeschichte. Dabei fungieren die Eindrücke, die der Dichter sofort produktiv verwertete, gewissermaßen als Strukturmomente des Textes, auf den sich dadurch letztlich die ,,tiefe und geheime' Ordnung“ des genannten Schneekristalls überträgt. Besonders anschaulich schildert Orhan den Kontext, in dem das Gedicht Schnee entstand, das dem Zyklus seinen Namen gibt:

Şiir az önce aklından aynı anda geçen pek çok şeyle yapılmıştı: Yağan kar, mezarlıklar, istasyon binasında neşeyle koşturan kara köpek, pek çok çoçukluk anısı ve otele dönüş yolunda adımları hızlandıkça mutluluk ve telaş arası bir duyguyla gözünün önünde canlanan İpek. (Pamuk, 2002, S. 90)

Das Gedicht war aus vielerlei Dingen gemacht, die ihm kurz zuvor gleichzeitig durch den Sinn gegangen waren: der fallende Schnee, Friedhöfe, der schwarze Hund, der fröhlich durch den Bahnhof tobte, zahlreiche Kindheitserinnerungen und Ipek, die er mit einem Gefühl, das zwischen Glück und Aufregung schwankte, vor Augen hatte, während sich seine Schritte auf dem Weg ins Hotel beschleunigten.

Neben Eindrücken, aus denen sich ein melancholisches Stimmungsbild von Kars zusammensetzt, und Erinnerungen an die Kindheit ist es besonders die Liebe zu İpek, die den Dichter beseelt. Doch werden seine Verse nicht nur von ihr, sondern allgemein von seinen sozialen Beziehungen zu den Karsern, diesen in einer verlorenen Stadt lebenden, Anderen', geprägt. Vor allem seine Gespräche mit den Quasi-Zwillingen Necip und Faz1l, deren Freundschaft in gewisser Weise seine Seelenverwandtschaft mit Orhan widerspiegelt (McGaha, 2008, S. 162), fließen - teilweise sogar wörtlich - in die Gedichte ein. So bringen diese letztlich, Pamuks Intention gemäß, „den Seelenzustand der Leute“ (Lau, 2005, S. 1) von Kars, d. h. ihre Ängste und Nöte, Hoffnungen und Träume, zum Ausdruck.

Beim „Schnee“ handelt es sich, wie gesehen, um eine besonders häufig vorkommende „Spur“ des Romans, und zwar nicht zuletzt deshalb, da sowohl der Gedichtzyklus des Protagonisten einschließlich des Titelgedichts als auch die Werkgeschichte des ,autodiegetischen Erzählers“ (bzw. der Roman des Autors) einschlägig betitelt sind. Dem differentiellen Spiel der hier gelegten „Spuren“ entspricht die enge Verweisstruktur, die zwischen Kas und Orhans Autorschaft besteht. Man kann sagen, dass ersterer von letzterem ersetzt wird. So erbringt der Erzähler letztlich eine doppelte Repräsentationsleistung: 
einerseits vertritt er den Autor im Text, andererseits den verstorbenen Protagonisten. An einer Stelle bezeichnet Orhan $\mathrm{Ka}$ und den ebenfalls verstorbenen Freund Fazils nicht zufällig als ,unsere[] leidenschaftlicheren, komplizierteren und authentischeren Originale“ (,daha tutkulu, daha karmaşık ve daha gerçek asıllarımız[]", Pamuk, 2002, S. 421).

Mit seiner stellvertretenden Autorschaft verfolgt der Romancier das paradoxe Ziel, das verschwundene Werk seines Freundes zu verewigen. Er versucht dies, indem er bestimmte Details, die bei der Entstehung der Gedichte eine Rolle spielten, in den Fokus rückt. Obwohl sich Ka jedoch „nie sehr mit Politik befaßt" (,siyasi sürgün hayatı yaşıyordu“, Pamuk, 2002, S. 10) und auch keine engagierte Literatur im engeren Sinne produziert hat, wird Schnee auf diese Weise zwangsläufig politisch. Tatsächlich eignet sich der Roman mit Theodor W. Adorno dafür, „,der sturen Antithese von engagierter und reiner Kunst zu Leibe zu rücken" (Adorno, 1974, S. 115). Dies verdankt sich Orhans dekonstruktiver Inversion, durch die der soziale Prozess sichtbar gemacht wird, aus dem Kas Werk letztlich hervorging. „Der Künstler, der das Kunstwerk trägt“, so Adorno, ,,ist nicht der je Einzelne, der es hervorbringt, sondern durch seine Arbeit, durch passive Aktivität wird er zum Statthalter des gesellschaftlichen Gesamtsubjekts“ (ebd. S. 126). Kars wird im Roman als eine von sozialem und wirtschaftlichem Elend heimgesuchte Stadt dargestellt. Zugleich aber handelt es sich hier in gewisser Weise um einen politischen Mikrokosmos, in dem sich „die türkische Wirklichkeit“ (,gerçek Türkiye[]“, Pamuk, 2002, S. 214) verdichtet. Das aus Ka und Orhan bestehende Tandem erscheint vor diesem Hintergrund als „Statthalter des gesellschaftlichen Gesamtsubjekts“ und als Fürsprecher der ,Anderen', mithin ,all derer, die sich kein Gehör verschaffen können“"(Pamuk, 2005, S. 27).

\section{Ausblick oder Spuren zu weiteren Werken Pamuks}

Zum Abschluss sei, wenngleich nur kurz und holzschnittartig, gezeigt, dass sich eine dekonstruktive Lesart, wie sie diesem Beitrag zugrunde liegt, prinzipiell auch auf andere Werke Pamuks anwenden lässt. Bezeichnenderweise enthält Schnee differentielle „Spuren“ zum Neuen Leben (Yeni Hayat, 1994), zu Rot ist mein Name (Benim Adım Kırmızı, 1998) und dem Museum der Unschuld (Masumiyet Müzesi, 2008). Erol führt sie im Einzelnen aus:

Like his previous novels, Snow also exhibits direct allusions to Pamuk's earlier works, for example to The New Life, which appears in this novel as the the name of a pastry shop. In Snow, Ka's encounter with İpek and others, as well as the murder oft he head oft he religious institute, all take place there. The limping dog wanders in from My Name Is Red and appears not only in Kars, but also in Frankfurt, two key locations in Snow. Pamuk even gives away the title of his next book, The Museum of Innocence, when Snow's narrator Orhan talks of the book he is currently writing. Clearly, Pamuk is deliberately flaunting the fact that each work is an extension of the previous ones. Growing out of each other, they are to be read as different manifestations of the problems he is most centrally interested in. (Erol, 2007, S. 409) 
Mit „Spuren“ dieser Art akzentuiert Pamuk, gemäß dem universalen Textverständnis Derridas, die eigenen Werke als tendenziell offen. Wenngleich Schnee hinsichtlich solcher Verweise eine Art Knotenpunkt darstellt, verläuft die einschlägige Struktur auch unabhängig von diesem Roman. Als Beispiel sei hier ein Fall angeführt, wie ihn Erol im Zitat für Schnee und Das Museum der Unschuld veranschlagt: Faruk Darvinoğlu, der „Enzyklopädiker“ aus dem Stillen Haus (Sessiz Ev, 1983) findet bei seinen Recherchen im Archiv in Gebze 1982 ein Manuskript mit dem Titel Steppdeckenmachers Stiefkind und veröffentlicht es mit einer Widmung an seine Schwester Nilgün, die an den Folgen einer von ihrem nationalistischen Cousin verübten Gewalttat gestorben ist. Einer der beiden Hauptprotagonisten in der zwei Jahre später erschienenen Weißen Festung aber, der Hodscha, ist der Sohn eines Steppdeckenmachers, der ihn in seiner Kindheit wie ein ungeliebtes Stiefkind behandelte. ${ }^{12}$

Dieser Roman handelt im Übrigen von der Geschichte des aus der Toskana stammenden „autodiegetischen“ Erzählers und eben jenes Hodschas, also zweier Repräsentanten des christlich geprägten Westens bzw. des islamisch geprägten Osmanischen Reiches. Schauplatz der Handlung ist das Istanbul des 17. Jahrhunderts. In einer den äußeren Umständen geschuldeten Herr- und Knecht-Beziehung betreiben der Hodscha und der Erzähler wissenschaftliche Forschungen sowie einen intensiven individuellen und religiös-kulturellen Austausch, der darin kulminiert, dass sie beschließen, ihre biografischen Identitäten $\mathrm{zu}$ wechseln und gleichsam das Leben des jeweils Anderen fortzuführen.

Die titelgebende Festung hat eine gewisse Ähnlichkeit mit dem bereits weiter oben zur Sprache gekommenen Schloss Kafkas: Sie ist uneinnehmbar und steht im Text symbolisch für die widersprüchliche Einstellung der Türken gegenüber dem Westen, mithin zur Unmöglichkeit, den kulturell wie religiös ,Anderen' zu verstehen. ${ }^{13}$ Ihr Name Doppio (,Doppel“, „doppelt“) verweist, wie unschwer zu

\footnotetext{
${ }^{12}$ Bei den genannten Beispielen handelt es sich um selbstreferenzielle Verweise, die insofern einer gesonderten Betrachtung wert sind, als Selbstreferenz in der Literaturwissenschaft - und nicht nur dort - einen eigenen Forschungsbereich bildet. Dass Pamuk dem „autodiegetischen“ Erzähler von Schnee seinen Namen gibt, unterstreicht nur, dass sich seine Texte für entsprechende Untersuchungen anbieten. Zudem spielt Selbstreferenz im Zusammenhang der Postmoderne eine wesentliche Rolle. Obwohl Pamuk im Allgemeinen der postmodernen Literatur zugeordnet wird, stellt die Dissertation von Saraçoğlu 2003 anscheinend die einzige Untersuchung dar, die sich explizit mit selbstreferenziellen Aspekten seines Werks befasst. Als Einführung in die Selbstreferenzforschung vgl. Luhmann 1993 a/b, Nöth 2000, Scheffel 1997, Jay 1984 und auch Hofstadter 1992.

${ }^{13}$ Dinge oder Werte, in denen sich die Vergeblichkeit bestimmter Bemühungen von Pamuk'schen Helden ausdrückt, Symbole der „différance“, wenn man so will, finden sich außer in Schnee und der Weißen Festung noch in anderen Pamuk-Romanen: In Cevdet und seine Söhne (Cevdet Bey ve Oğullarl, 1982) sowie im Stillen Haus sind es westliche Ideale, die einzelne Figuren vergeblich zu verwirklichen versuchen, im Neuen Leben ist es der Lichtkegel und in Rot ist mein Name die Farbe Rot.
} 
erkennen ist, auf das „Spiel der Differenzen“, das auch in diesem Roman Pamuks betrieben wird.

Bei den genannten Punkten handelt es sich - wie bei so vielem im vorliegenden Beitrag - um beliebig fortführbare Beispiele, die zeigen, dass Pamuks gesamtes Werk zu einer dekonstruktiven Analyse einlädt.

\section{BIBLIOGRAPHIE}

\section{Primärliteratur}

Kafka, F. (1995). Das Schloß. Roman in der Fassung der Handschrift. In: Gesammelte Werke in zwölf Bänden. Nach der Kritischen Ausgabe, hrsg. von Hans-Gerd Koch. Bd. 4. Frankfurt a.M.: Fischer Taschenbuch Verlag.

Mann, T. (1960). Der Zauberberg. Roman. In: Gesammelte Werke in zwölf Bänden. Band III. Frankfurt a.M.: S. Fischer Verlag.

Pamuk, O. (1998). Das neue Leben. (Übers. von Ingrid Iren). München/Wien: Carl Hanser Verlag. - (2001). Rot ist mein Name. (Übers. von Ingrid Iren). München/Wien:

Carl Hanser Verlag. (2002). Kar. Istanbul: Iletişim.

------- (2005a). Die weiße Festung. (Übers. von Ingrid Iren). München/Wien: Carl Hanser Verlag.

(2005b). Eine Schule des Verstehens. Dankesrede zum Friedenspreis des deutschen Buchhandels. 2005 Börsenblatt-FRIEDENSPREIS.

------ (2005c). Schnee. (Übers. von Christoph K. Neumann). München/Wien: Carl Hanser Verlag.

(2008). Das Museum der Unschuld. (Übers. von Gerhard Meier). München/Wien: Carl Hanser Verlag.

---------- (2009). Das stille Haus. (Übers. von Gerhard Meier). München/Wien: Carl Hanser Verlag.

(2011) Cevdet und seine Söhne. (Übers. von Gerhard Meier). München/Wien: Carl Hanser Verlag.

\section{Sekundärliteratur}

Adorno, T. W. (1974). Der Artist als Statthalter. In: Noten zur Literatur I. Frankfurt a.M.: Suhrkamp Verlag.

Birus, H. (Hg.). (1982). Hermeneutische Positionen: Schleiermacher - DiltheyHeidegger - Gadamer. Göttingen: Vandenhoeck \& Ruprecht Unipress.

Blumenberg, H. (1997). Paradigmen zu einer Metaphorologie. Frankfurt a. M.: Suhrkamp Verlag.

Brod, M. (1968). Nachwort zur ersten Ausgabe. In: Kafka, Franz: Das Schloß. Frankfurt a.M./Hamburg: Fischer Bücherei.

Clemens, C. A. L. (2011). Suicide Girls: Orhan Pamuk's Snow and the Politics 
of Resistance in Contemporary Turkey. In: Feminist Formations, Vol. 23, Issue 1. The Johnns Hopkins University Press, 138-154.

Culler, J. (1988). Dekonstruktion. Derrida und die poststrukturalistische Literaturtheorie. Reinbek bei Hamburg: Rowohlt Taschenbuch Verlag GmbH.

Derrida, J. (1976). Die Schrift und die Differenz. Frankfurt a. M.: Suhrkamp Verlag.

(1983). Grammatologie. Frankfurt a. M.: Suhrkamp Verlag.

(1990). Semiologie und Grammatologie. Gespräch mit Julia Kristeva. In: Postmoderne und Dekonstruktion. Texte französischer Philosophen der Gegenwart, hrsg. von Peter Engelmann. Stuttgart: Philipp Reclam jun.

Erol, S. (2007). Reading Orhan Pamuk's Snow as Parody: Difference as Sameness. In: Comparative Critical Studies. Vol. 4, Issue 3. Edinburgh: Edinburgh University Press, 403-432.

Genette, G. (1998). Die Erzählung. München: Wilhelm Fink Verlag.

Göknar, E. (2013). Orhan Pamuk, Secularism and Blasphemy. The Politics of the Turkish Novel. London/New York: Routledge.

Hiebel, H. H. (1999). Franz Kafka: Form und Bedeutung. Formanalysen und Interpretationen von Vor dem Gesetz, Das Urteil, Bericht für eine Akademie, Ein Landarzt, Der Bau, Der Steuermann, Prometheus, Der Verschollene, Der Proce $\beta$ und ausgewählten Aphorismen. Würzburg: Verlag Königshausen und Neumann.

Hofstadter, D. R. (1992). Gödel, Escher, Bach, ein Endloses Geflochtenes Band. München: Deutscher Taschenbuch Verlag.

Jay, P. (1984). Being In The Text: Self-Representation From Wordsworth to Roland Barthes. Ithaca: Cornell University Press.

Karthaus, U. (1983). Der Zauberberg (1924). In: Deutsche Romane des 20. Jahrhunderts. Neue Interpretationen, hrsg. von Paul Michael Lützeler. Königstein/Ts.: Athenäum.

Koopmann, H. (1983). Der klassisch-moderne Roman in Deutschland. Thomas Mann, Alfred Döblin, Hermann Broch. Stuttgart (u.a.): Kohlhammer Verlag.

Lau, J. (2005). Achte auf die Details des Lebens. Letzter Zugriff: 16.04.2013. www.zeit.de/2005/16/InterviewPamuk

Luhmann, N. (1993a). Soziale Systeme: Grundriß einer allgemeinen Theorie. Frankfurt a. M.: Suhrkamp Verlag.

(1993b). Zeichen als Form. In: Kalkül der Form, hrsg. von Dirk Baeker. Frankfurt a. M.: Suhrkamp Verlag.

McGaha, M. (2008). Autobiographies of Orhan Pamuk. The Writer in His Novels. Salt Lake City: The University of Utah Press.

Mehnaz, M. A. \& David, M. B. (2012). Global Perspectives on Orhan Pamuk. 
Existentialism and Politics. New York: Palgrave Macmillan.

Mennicken, P. (2002). Für ein ABC des Menschenbenehmens. Menschenbild und Universalethos bei Thomas Mann. Mainz: Matthias-Grünewald-Verlag.

Neumann, J. (2011). Religious Cosmopolitanism? Orhan Pamuk, the Headscarf Debate and the Problem with Pluralism. In: Minnesota Review No 77. Minessota: Duke University Press, 143-161.

Nöth, W. (2000). Handbuch der Semiotik. Stuttgart: J. B. Metzler Verlag.

Saraçoğlu, S. (2003). Self-Reflexivity in Postmodernist Texts: A Comparative Study oft the Works of John Fowles and Orhan Pamuk. Ankara: Orta Doğu Teknik Üniversitesi Sosyal Bilimler Enstitüsü.

Scheffel, M. (1997). Formen selbstreflexiven Erzählens. Eine Typologie und sechs exemplarische Analysen. Tübingen: Niemeyer Verlag.

Schmidt, C. (1997). „Ehrfurcht und Erbarmen“. Thomas Manns NietzscheRezeption 1914 bis 1947. Trier: Wissenschaftlicher Verlag Trier.

Shu-chun, C. C. (2015). Unveiling the Veil: Headscarf and Identity Crises in Orhan Pamuk's Snow. Letzter Zugriff: 14.06.2015. http://zephyr.nsysu.edu.tw/researchcenter2/document/snow\%2002-headscarf.pdf

Tholen, T. (1999). Erfahrung und Interpretation. Der Streit zwischen Hermeneutik und Dekonstruktion. Heidelberg: Universitätsverlag Winter.

Zima, P. V. (1994). Die Dekonstruktion. Einführung und Kritik. Tübingen und Basel: Francke Verlag.

\title{
GENESIS OF A LOST MANUSCRIPT: ORHAN PAMUK'S SNOW FROM A DECONSTRUCTIVE PERSPECTIVE
}

\begin{abstract}
Deconstruction has hardly ever been applied as a method to the analysis of Orhan Pamuk's Snow, which is surprising because the novel itself is deconstructive to some extent. The following article addresses this issue by providing a deconstructive view of the text according to the approach of Jacques Derrida. The paper starts with a short account of Derrida's understanding of texts as universal "differences and traces of traces". This perspective is exemplified with the aid of the metaphor for snow and Franz Kafka's Castle. In connection with the latter, Derrida's thoughts about "différance", which thwarts the presence of meaning, are explained as well. The following section addresses Pamuk's depiction of a "suicide epidemic" which is combined with the complex issue of headscarf. The attempts of the protagonist $\mathrm{Ka}$ to discover the reasons of the suicides are futile because, as will be shown, individual cases are affected by postponements of meaning, which are related with the notion of "différance". Understanding is also a problem for the narrator Orhan. The hypothesis developed in the concluding section of the analysis is that he is in fact narrating a deconstructive genesis of a lost manuscript. The collection of Ka's poems titled Snow, which Pamuk's novel is about for the most part, has disappeared. Meanwhile, the replacement of the poems
\end{abstract}


with informations about the circumstances of their creation makes the contextual socio-cultural problematics apparent.

Keywords: Orhan Pamuk, Snow, Franz Kafka, Deconstruction, Différance, Understanding, Genesis of a Lost Manuscript. 